\title{
Lichen Planopilaris in the Setting of Hair Sunscreen Spray
}

\author{
Theresa N. Canavan ${ }^{\mathrm{a}}$ Sarah F. McClees ${ }^{\mathrm{b}}$ James Robert Duncan ${ }^{\mathrm{a}}$ \\ Boni E. Elewski ${ }^{a}$ \\ ${ }^{a}$ Department of Dermatology, University of Alabama Birmingham, Birmingham, AL, USA; \\ bSchool of Medicine, University of Alabama Birmingham, Birmingham, AL, USA
}

\section{Established Facts}

- Frontal fibrosing alopecia is a type of scarring alopecia that presents on the frontal hairline and has recently been associated with regular facial sunscreen use in both men and women. The etiology of lichen planopilaris is unknown and has not been linked to sunscreen use.

\section{Novel Insights}

- Here we report a case of lichen planopilaris developing in the hair part after long-term application of spray-on sunscreen to the hair part line.

\section{Keywords}

Lichen planopilaris · Frontal fibrosing alopecia · Benzyl salicylate $\cdot$ Sunscreen $\cdot$ Scarring alopecia

\begin{abstract}
Lichen planopilaris (LPP) is a scarring alopecia seen classically in older Caucasian women. Frontal fibrosing alopecia (FFA) is a distinct disease that shares the histologic and trichoscopic features of LPP but differs in its clinical presentation in that it involves the frontal scalp and occasionally the eyelashes and eyebrows of older Caucasian women. Several recent studies have described a link between FFA and the use of sunscreen. Here we report a case of LPP aris-
\end{abstract}

\section{KARGER}

(c) 2018 S. Karger AG, Basel

E-Mail karger@karger.com

www.karger.com/sad ing in the part line of the scalp of a woman with a history of long-term daily application of spray-on sunscreen to the hair part line.

(c) 2018 S. Karger AG, Basel

\section{Introduction}

Lichen planopilaris (LPP) is an uncommon scarring alopecia of unknown etiology that typically presents as patchy hair loss on the scalp of women. Frontal fibrosing alopecia (FFA) is a distinct disease that shares the histologic and trichoscopic features of LPP but differs in its clinical presentation in that it involves the frontal scalp 


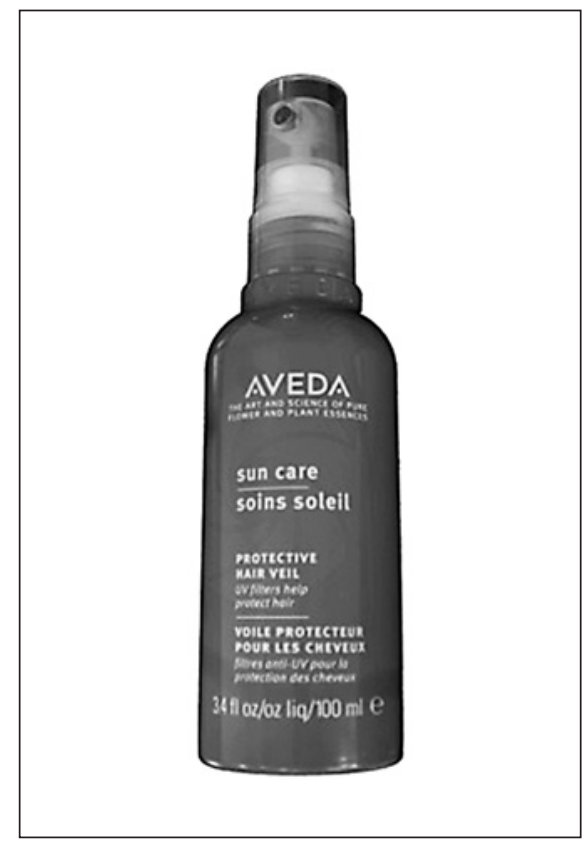

Fig. 1. Spray-on scalp sunscreen used regularly by patient prior to the development of her lichen planopilaris.

and occasionally the eyelashes and eyebrows of older Caucasian women [1]. The incidence of FFA has increased over the past decade, and its development has recently been linked to regular sunscreen use [2-4].

Here we report a case of LPP developing in the part line of the scalp after extended use of spray-on scalp sunscreen, suggesting a possible association between sunscreen use and the development of LPP in select patients.

\section{Case Report}

A 56-year-old Caucasian female presented to our Universitybased outpatient dermatology clinic with a 6-month history of progressive hair loss and moderate scalp pruritus. On exam there was diffuse ill-defined erythema of the hair part line and surrounding scalp. Trichoscopy revealed a decreased number of follicular ostia, perifollicular scale, and follicular white plugging along the hair part line. Eyelashes and eyebrows were intact. A punch biopsy was performed along the active edge of alopecia. Histologically there was a dense dermal band-like lymphocytic infiltrate at the level of the infundibulum and isthmus along with scattered necrotic keratinocytes within the follicular epithelium. There was minimal dermal fibrosis, no increased dermal mucin, and the catagen/telogen ratio remained intact. She was given a diagnosis of early LPP based on the clinical and histologic findings.

The patient has since been followed in our department for over 3 years. She was initially treated with hydroxychloroquine $200 \mathrm{mg}$ twice daily, biotin $5 \mathrm{mg}$ daily, and topical corticosteroids, which halted the progression of her hair loss. She was also advised to avoid applying any fragrance-containing products to the scalp but was not compliant with this recommendation. Unfortunately, she developed visual changes which led her to discontinue hydroxychloroquine. She subsequently experienced a flare in her disease and was then started on pioglitazone $15 \mathrm{mg}$ daily and uptitrated to $30 \mathrm{mg}$ daily leading to another remission of her LPP. The patient self-discontinued pioglitazone once her disease was well controlled and experienced a new flare. She was once again restarted on pioglitazone $15 \mathrm{mg}$ daily along with topical corticosteroids, which brought her disease under control.

The patient recently revealed to us that for the past several years she had been applying a spray-on sunscreen daily to her hair part line, which has been the area where she has had the most prominent disease activity. She also noted that she has parted her hair on the same side of her scalp since before she started using the sunscreen. The product was Sun Care Protective Hair Veil, produced by Aveda (Fig. 1), which contains benzyl salicylate as a chemical UV-blocking ingredient. We recommended that she discontinue this product. The patient was continued on pioglitazone with the plan to stop at 1 year following remission and has since been lost to follow-up.

\section{Discussion}

LPP is a scarring alopecia that classically affects the scalp of women. It is characterized clinically by follicular plugging and perifollicular scale and erythema, which are best visualized by trichoscopy [5]. Histologic findings include a lichenoid lymphocytic infiltrate and progressive dermal fibrosis. The disease is often chronic and progressive, and achieving remission is often challenging [1].

While the precise etiology of LPP remains elusive, FFA has recently been associated with regular sunscreen exposure $[3,4,6]$. FFA is indistinguishable from classic LPP on trichoscopy and histology, but it has a distinct clinical presentation as a band-like area of alopecia involving the frontal and frontotemporal scalp in postmenopausal Caucasian women. There is also occasional involvement of the eyebrows and eyelashes [1].

The incidence of FFA has been increasing over the past decade. FFA, which was initially described in 1994 and remained rare during the 1990s, is now a commonly encountered primary cicatricial alopecia with increasing incidence across many countries $[2,5,7]$. In a recent survey-based study in the United Kingdom, FFA was one of the most commonly encountered primary cicatricial alopecias by British dermatologists [8].

While the rising incidence of FFA may be due to increased awareness and reporting, this trend also suggests a possible environmental exposure as a trigger $[3,6,9]$. Recent surveys of both men and women have noted sta- 
tistically significant associations between regular facial sunscreen use and FFA prevalence. Female FFA patients were twice as likely to report regular application of facial sunscreen compared to non-FFA controls ( $48 \%$ vs. $24 \%$ ) [6]. Even more striking is the association reported in men: a recent survey documented high rates of regular facial sunscreen use in male FFA patients relative to non-FFA male controls ( $71 \%$ vs. 11\%) [3]. However, these types of studies are subject to recall bias. Additionally, sunscreen is not exclusive to facial products. A review of 60 random hair-care products found that a majority of leave-on and wash-off hair products contained a sunscreen chemical, and a survey in one clinic revealed use of leave-on haircare products was high in both patients with FFA (81\%) and LPP (70\%). It has been suggested that if sunscreen chemicals do play a role in the development of FFA, the presence of UV filters in hair-care products could explain the occasional progression of FFA to wider scalp involvement [4].

Our patient reported a long history of regular application of spray-on sunscreen to the exposed portion of her scalp at the hair part line, which was the area affected by her LPP. This is the first report describing the development of LPP in the setting of spray-on scalp sunscreen use. Just as the distribution of FFA seems to match the usual sites of facial sunscreen application, the distribution of our patient's LPP parallels the site of her spray-on sunscreen use. While this relationship may be coincidental, recent reports suggest an association between facial sunscreens and FFA. It could be argued that the areas involved are exposed to the sun, and perhaps the association is not with the sunscreen, but with sun exposure.
However, it is conceivable that our patient's spray-on sunscreen use may have had a role in the development of her LPP.

The association between regular sunscreen use and the risk for developing FFA is intriguing, especially in light of the increasing incidence of FFA worldwide. Although FFA and LPP are distinct diseases with unique clinical presentations, their identical trichoscopic and histologic features would suggest that similar environmental factors could play a role in their pathogenesis. Further cross-sectional studies are needed to establish the relationship between sunscreen use and LPP. Specifically, studies examining the use of spray-on sunscreen applied to the scalp as well as those comparing chemical versus physical blocking sunscreens will be of particular interest.

\section{Statement of Ethics}

Proper consent was obtained from the included patient.

\section{Disclosure Statement}

No external or institutional funding was received for this study. This study has not been published or presented elsewhere in any form. Conflicts of interest: Dr. Boni E. Elewski: Clinical Research Support (research funding provided to the University of Alabama Birmingham): Abbvie, Boehringer Ingelheim, Celgene, Incyte, Leo, Lilly, Merck, Novartis, Pfizer, Regeneron, Sun, Valeant (Ortho dermatology). Consultant (received honorarium): Boehringer Ingelheim, Celgene, Leo, Lilly, Novartis, Pfizer, Sun, Valeant (Ortho dermatology). None of the above disclosed conflicts are relevant to this paper. The remaining authors have no conflicts of interest to disclose.

\section{References}

1 Weston G, Payette M: Update on lichen planus and its clinical variants. Int J Womens Dermatol 2015;1:140-149.

2 Miteva M, Whiting D, Harries M, Bernardes A, Tosti A: Frontal fibrosing alopecia in black patients. Br J Dermatol 2012;167:208-210.

3 Debroy Kidambi A, Dobson K, Holmes S, Carauna D, Del Marmol V, Vujovic A, et al: Frontal fibrosing alopecia in men: an association with facial moisturizers and sunscreens. Br J Dermatol 2017;177:260-261.
4 Callander J, Frost J, Stone N: Ultraviolet filters in hair-care products: a possible link with frontal fibrosing alopecia and lichen planopilaris. Clin Exp Dermatol 2018;43:69-70.

5 Inui S, Nakajima T, Shono F, Itami S: Dermoscopic findings in frontal fibrosing alopecia: report of four cases. Int J Dermatol 2008;47: 796-799.

6 Aldoori N, Dobson K, Holden CR, McDonagh AJ, Harries M, Messenger AG: Frontal fibrosing alopecia: possible association with leave-on facial skin care products and sunscreens; a questionnaire study. Br J Dermatol 2016;175:762-767.
7 Dlova NC, Jordaan HF, Skenjane A, Khoza N, Tosti A: Frontal fibrosing alopecia: a clinical review of 20 black patients from South Africa. Br J Dermatol 2013;169:939-941.

8 Griffin LL, Michaelides C, Griffiths CEM, Paus R, Harries MJ: Primary cicatricial alopecias: a U.K. survey. Br J Dermatol 2012;167: 694-697.

9 Seegobin SD, Tziotzios C, Stefanato CM, Bhargava K, Fenton DA, McGrath JA: Frontal fibrosing alopecia: there is no statistically significant association with leave-on facial skin care products and sunscreens. Br J Dermatol 2016;175:1407-1408. 